\title{
Design of a New Type of Test System for State of Power Battery LV Li Ya ${ }^{1, a}$, MIN Yong Jun ${ }^{1, b}$, WANG Xin Can $^{1, c}$ \\ ${ }^{1}$ College of Automobile and Traffic Engineering, Nanjing Forestry University, Nanjing, China alvliya@163.com, ${ }^{b}$ yjmin@njfu.com.cn, caboutwxc@163.com
}

Keywords: storage battery; observation system; LabVIEW

\begin{abstract}
In the vehicle energy management and battery management system, the parameter monitoring system of the battery is the important basis to ensure its operation. The new observation system for state of battery is designed according to the principle of storage battery and its charge and discharge characteristics. It is also combined with the related hardware , basing on LabVIEW software and communication of GPIB bus. The system realizes high precision data acquisition and analysis of power battery's voltage, electric current, real-time temperature and other dynamic information. The experimental results show that the detection system designed is stable and it conforms to requirements of storage battery's detection. It improves the convenience and accuracy of storage battery's measurement.
\end{abstract}

\section{Introduction}

Power battery as one of the important power source of electric vehicles, its performance directly affects the car starting, acceleration, mileage, etc. State information of power battery is the vehicle energy management system for power distribution and an important basis for battery management. Accurate battery status information for efficient management of the battery and the vehicle performance improvement has important significance. Therefore, for convenience of automatic monitoring of power battery is the important content of the electric car research and development ${ }^{[1-2]}$. With the development of the LabVIEW software technology and its flow chart of graphical programming and multithreading running control technology, to the power battery monitoring system provide a good software development platform. Compared with instrument direct measurement of single inconvenience, in this paper, using the computer instead of instrument operation, and use the high precision acquisition and visual display of the LabVIEW function ${ }^{[3]}$, based on GPIB protocol and the corresponding hardware, design a new type of power battery condition monitoring system, realize to monitor power battery dynamic parameters conveniently.

\section{Power battery measurement system hardware design}

To achieve the effective measurement of the parameters of the battery status, we constructed the system hardware diagram as shown in Fig. 1. The system hardware structure contains lithium ion power battery module, multimeter module, power module, electronic load module, GPIB acquisition controller and human-computer interaction platform ${ }^{[11-12]}$.

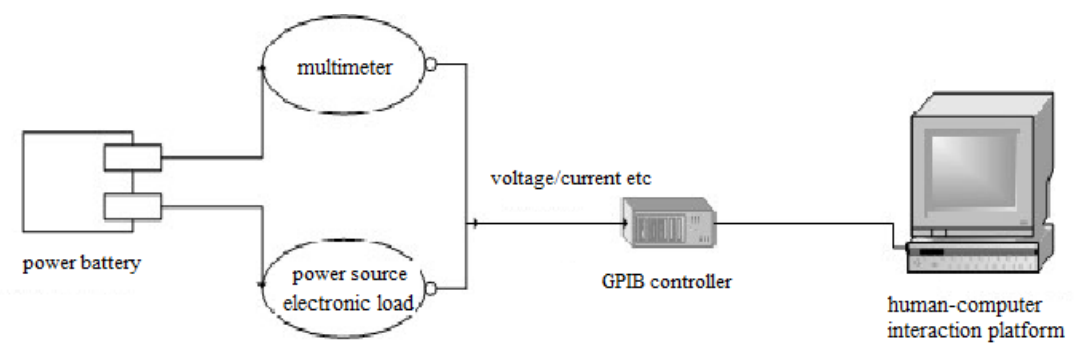

Fig.1 Block Diagram of Hardware System

GPIB controller contains the IEEE488 protocol, through the human-computer interaction platform can be real-time control instrument with GPIB interface ${ }^{[4]}$. Controllers are connected to the computer at work, GPIB data records in computer hard disk, can also be recorded in the SD card 
at the same time. The battery temperature sensor are installed in GPIB acquisition controller, can measure the real-time temperature of the battery

During the test the GPIB acquisition controller and multimeters, power supplies and PC connected. When the power battery charging, multimeter can real-time display of voltage, and at the same time power equipment display of voltage and current, when need can also be used for low power battery discharge, GPIB acquisition controller based on IEEE488 protocols transmit the data to the PC. When the battery discharge, the electronic load can pass data to PC through GPIB protocols; Processing the data through the PC and shows the battery voltage and current waveform and their parameters, such as curve graph, temperature sensor for power battery temperature measurement and passed to the PC.

\section{The LabVIEW software design}

\section{VISA serial communication module}

VISA is used for virtual instrument system of standard API, itself does not have the ability to programming and it by calling the underlying driver implementation of instrument programming ${ }^{[5}$, $\left.{ }^{13}, 14\right]$. Because the VISA has unified mechanism of management, operation and use of equipment resources, and provides the powerful instrument control function, so the system can be sent via VISA SCPI command to control GPIB instruments, and reads the GPIB protocol transfer the data to the PC.

System adopts COM4 as a serial port resource interface, using the VISA function receives the data, First use of VISA configuration function for a serial port initialization, and configure the relevant parameters; Then VISA read function and write a function to read and write operations, data communication in the process of sending or receiving data are strings. When the program is in the state of the test, the instrument with VISA write and read function of address and display the data to the PC, with the VISA Close-function Close VISA resources and release all resources associated with it.

\section{The state machine design}

Each parameter measurement need to be by changing the different status in the state machine to implement. In LabVIEW, any a state machine is made up of three basic parts, the first part is a while loop, the while loop is used to maintain the operation of the state machine, the second part is contained in the conditions of the while loop structure, used to determine a different state, the third part is the shift register, used to cede a state to a state judgment ${ }^{[6]}$ in the next cycle. Specific state diagram design is shown in Fig.2.

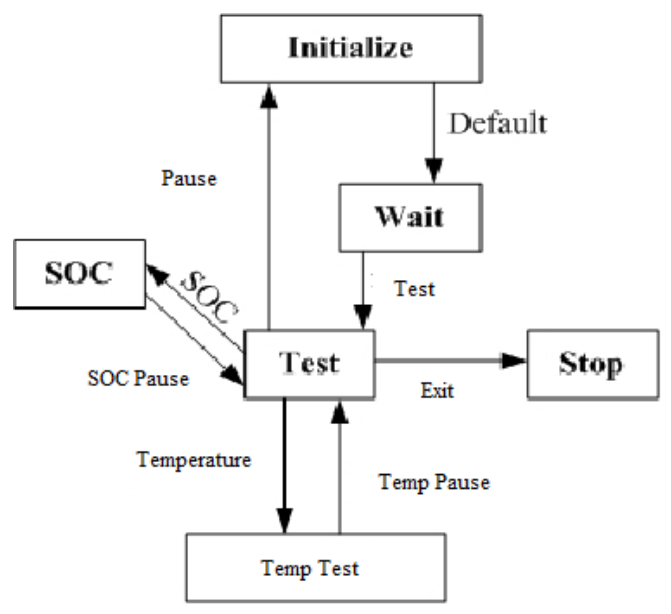

Fig.2 Diagram of System Status

\section{1)The default state of the state machine}

In the default state machine, the use of VISA function to set the communication parameters of transmission baud rate, numerical bits. Send the SCPI command to get type information of a measuring instrument, and read the address of the instrument. And through the transition function 
conversion between numeric and string, communication to ensure accurate and correct. When the system starts running, the program enters the default state, began to parse the VISA function related parameters and reads the instrument. The main program block diagram is shown in Fig.3.

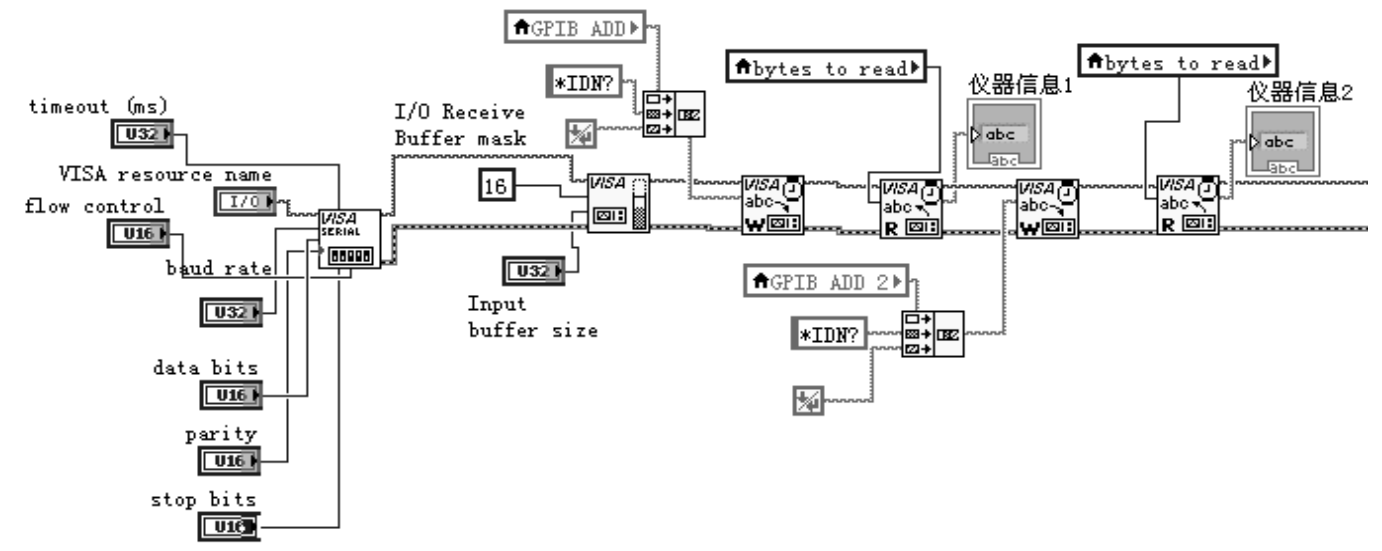

\section{2)The state machine test status}

Fig.3 Program Block Diagram of Default State

Test condition and structure is divided into two conditions, one is the temperature test program structure, the other is the current integral program structure. This structure contains two conditions of voltage and current data acquisition module, is to prevent temperature measurement or battery SOC (State of charge) test influence power battery parameters of data acquisition, which affects the measurement of the whole system.

Fig.4 is the main program, which consists of voltage, current and temperature monitoring module. Power battery charging and discharging requires the right temperature environment, so the power battery temperature acquisition and analysis is of great significance. System in real-time temperature measurement, power battery charge and discharge of dc voltage and dc current measurement without interference. So by this software system can achieve the real time monitoring, power battery charge and discharge can be judged whether battery equalization method is proper, without the need to monitor by measuring instrument.

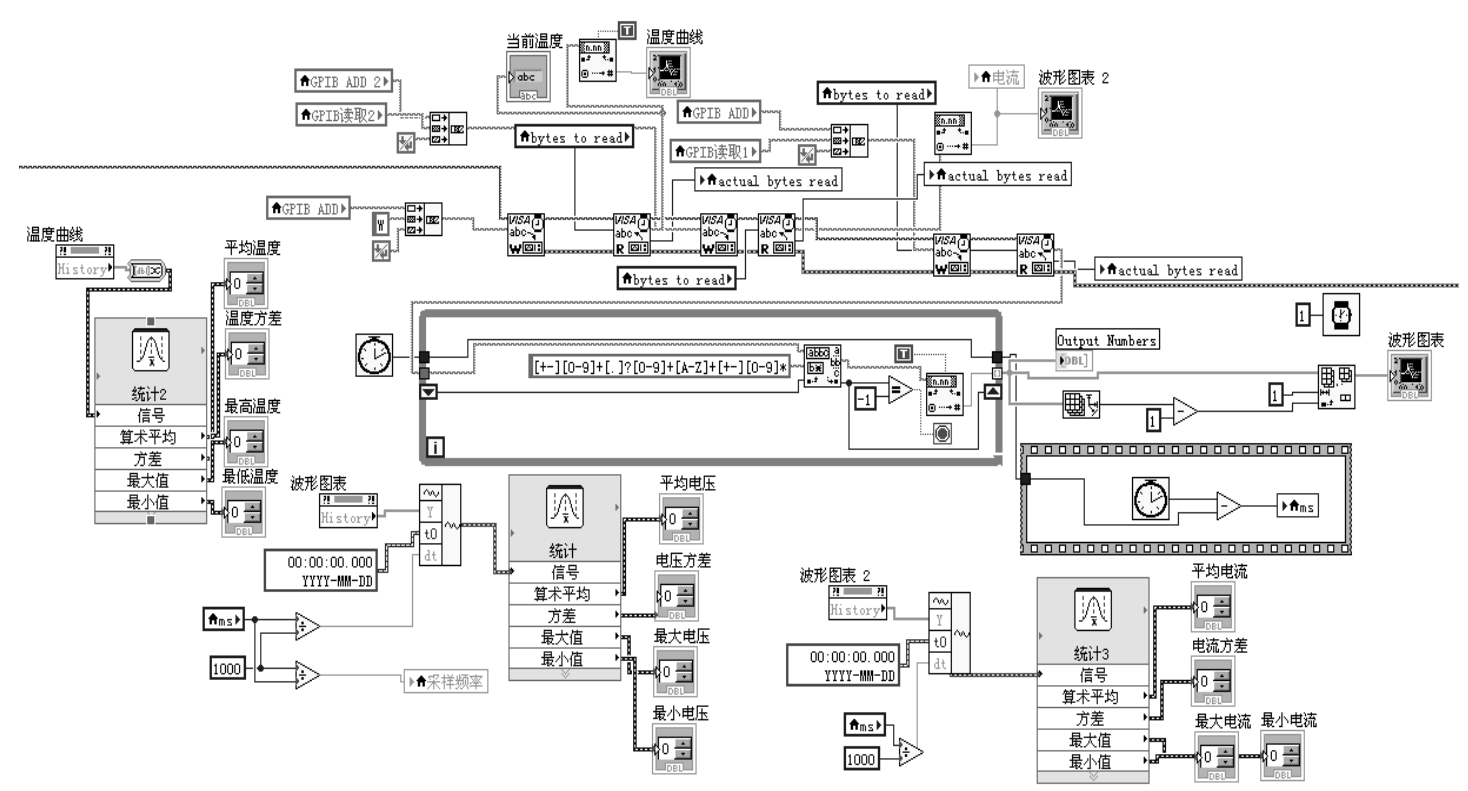

\section{Current integral module}

Fig.4 Block Diagram of Main Program

System can not only for power battery voltage, current and temperature monitoring, also using the current integral method for estimation of power battery SOC. Application system using A-H measuring principle, the programming block diagram, for integrating current collected, as shown in Fig. 5. Waveform chart 2 is current numerical display, through the attribute node to current value 
passed to the "create wave function", at the same time, the sampling frequency and other necessary data collection, unified to the integral function to calculate and use the waveform chart for display. Through improving the measuring accuracy of current, and then considering influence factors such as charge and discharge efficiency, can enhance the accuracy of SOC estimation ${ }^{[7]}$.

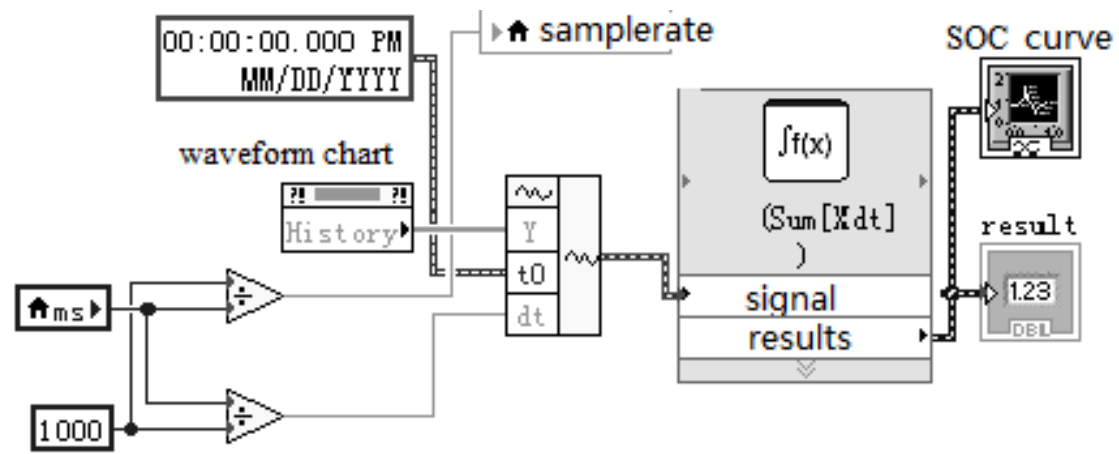

Fig.5 Program Block Diagram of Current Integration

\section{System test platform and display}

Monitoring system experimental platform is shown in Fig. 6. The top is a human-computer interaction platform, the left side of the middle layer is high precision programmable multimeter, the right side is the ithiumion power battery pack, the power supply and electronic load is on the bottom layer.

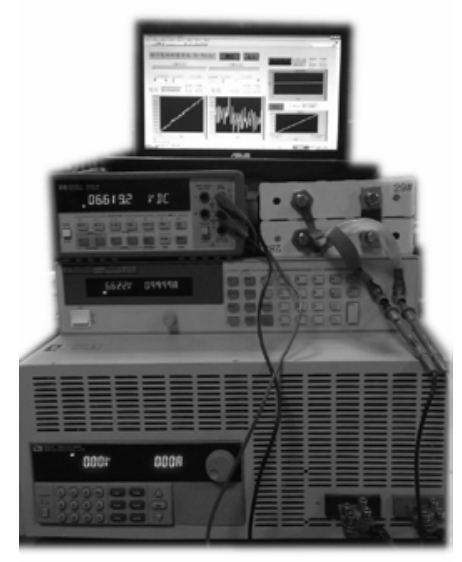

Fig.6 Test Platform of Monitoring System

LabVIEW front panel is the human-computer interaction interface, as shown in Fig. 7. Interface can display dynamic information of the battery, and the operation of the control program [8]. The front panel is divided into three modules, respectively is power battery voltage and current collection and display module, temperature acquisition module and the current integral module. When click the run button, the system will automatically read instrument model and displayed, and enter to the default state. Button corresponding to the different testing requirements, for example, click on the SOC button the system automatically for integral calculation, the current collected and displays real-time values.

All the procedures of operation, including voltage, current, temperature and SOC data collection and analysis, in a while loop in the body, can always run, until you click "exit" button, the program will stop running. 


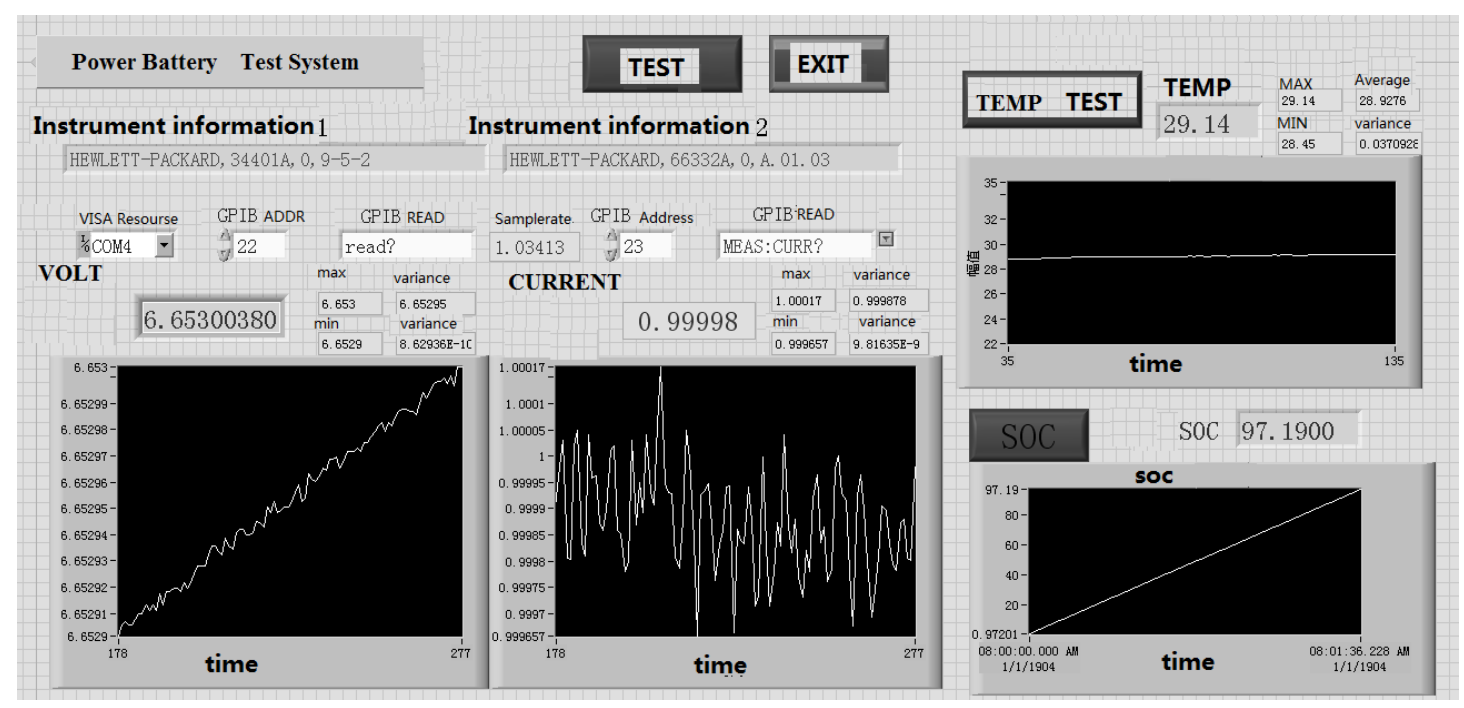

Fig.7 Interface Display of Front Panel

Modern GPIB instruments, basically support the SCPI language standard, to a variety of instruments, the benefits of using it is the basic control commands is the same, that is why the design SCPI command. General programmable instrument has its own set of commands, can be found in the documents, so the use of GPIB protocol can easily control the different types of instruments, achieve different measurement functions, convenient software function extension, achieve the function of a variety of tests.

\section{Conclusions}

In this paper we design a new type of power battery status monitoring system. Collection using GPIB controller and temperature sensors, combined with other corresponding measuring instrument, through GPIB bus to achieve data acquisition and recording, and the collected data sent to the PC monitoring software. Using LabVIEW to write the power battery voltage, current, temperature data acquisition and display program. The measurement and control system can measure information collection on display in an interface, realized the battery voltage, current, temperature and SOC value parameters real-time dynamic measurement, and can analyze the battery charge and discharge parameters and control. Actual test shows that the design of monitoring system is stable and precise data sampling, can accurately real-time dynamic monitoring of power battery.

\section{References}

[1] Junwen Zhou, Xiangwen Zhang. Power battery state parameter monitoring system for the design and implementation of [J]. Electric measurement and instrumentation, 2014,51 (16): 112-116.in Chinese.

[2] Zhihao Yu, Linjing Xiao, Long Chang, etc. The design of the lithium ion battery power supply current detection system [J]. Power supply technology, 2014,38(4):640-643. in Chinese.

[3] Xiaopan Xu,Jianzhao Zhou. Engineering equipment noise measurement system based on LabVIEW and sound design [J]. Journal of mechanical manufacturing and automation, 2014,41 (2):134-136. in Chinese.

[4] Hong He, Jinzhou Zhang, Zhihong Zhang. Based on GPIB interface of data acquisition system design [N]. Journal of tianjin university of technology, 2014,30(1):30-33. in Chinese.

[5] Zhanjun Tang, Lihui Feng, Bin zhang. The design and implementation of intelligent instruments based on virtual instrument [J]. Microcomputer information, 2008, 24 (1) : 252-254. in Chinese.

[6] Shuxue Chen,Xuan Liu. LabVIEW treasure dian [M]. Beijing: electronic industry press, 2011: 391-395. in Chinese. 
[7] Jinchao $\mathrm{Xu}$. Real-time on-line monitoring system for electric vehicle power battery and SOC estimation [D]. Guangdong, guangdong university of technology, 2014:8 -13. in Chinese.

[8] Xiping Pei,Hong, Xiao.Current detection in intelligent motor protector [J]. The design and implementation of instrument technique and sensor, 2012, 2:63-65. in Chinese.

[9] Barcena Soto, Casillas,Garcia Torales,et al.Discharge curves for a Ni-Cd secondary battery monitored by LabVIEW[J]. Revista Mexicana de Física, 2005(51):132.

[10] Banaeia, Khoobroo A, Fahimi B. Online detection of termi- nal voltage in voltage in Li-ion batteries via battery impulse respone[C]//2009 IEEE Vehicle Power and Propulsion Conference. US:IEEE, 2009:194-198.

[11] Valer P,Henk J B,Dmitry D.Battery Management Systems:Accurate Stata-of-Charge Indication for Battery-Powered Applications [M]. Germany:Springer, 2008.

[12] Szumanowski A,Chang Y H.Battery Management System Based on Battery Nonlinear Dynamics Modeling[J]. IEEE Transaction on Vehicular Technology,2008,57(3):1425-1432.

[13] Vladimir Zdornov,Yitzhak Birk.Battery and energy mangement in fleets of switchable battery EVs[J].Innovative Smart Grid Technologies,2011(2): 1-7.

[14] Jason Wynne. Impact of plug-in hybrid electric vehicles on California's electricity grid[D]. North Carolina: Duke University, 2009. 